\title{
Rehabilitación integral de barriadas con dificultades sociales en Andalucía. La experiencia de San Martín de Porres en Córdoba
}

\section{Integral renovation of districts with social difficulties in Andalusia. The experience of San Martín de Porres in Córdoba}

E. Morón Serna ${ }^{(*)}$, J.I. Rodríguez Galadí ${ }^{* *}$

\section{RESUMEN}

La recuperación de barriadas construidas en la segunda mitad del siglo xx, especialmente aquellas con mayores dificultades sociales, constituye una alternativa sostenible al fracaso del continuo crecimiento urbano. Es necesario promover actuaciones integrales que corrijan el deterioro y carencias de edificios residenciales, mejoren las relaciones de estos ámbitos con sus entornos urbanos, los doten de equipamientos y espacios públicos de calidad e impulsen la participación vecinal y su desarrollo social y económico. A partir de experiencias e instrumentos desarrollados en la última década en Andalucía, se analiza la rehabilitación integral de la barriada de San Martín de Porres en Córdoba. Se aborda su desarrollo urbanístico y arquitectónico -donde sobresale la figura de Rafael de La-Hoz- y las transformaciones sociales de su población. Del diseño, gestión y ejecución de la experiencia analizada se destacan los aspectos más relevantes, con el objetivo de facilitar su consideración en las futuras políticas de rehabilitación.

Palabras clave: Barriadas; rehabilitación integral; ciudad; social; Córdoba; Rafael de La-Hoz.

\section{ABSTRACT}

The recovery of the districts built during the second half of the twentieth century, especially those with greater social difficulties, constitutes a sustainable alternative to the failure of continuous urban growth. It is essential to promote integral actions in order to correct both deficiencies and deterioration in residential buildings, improve the relationship of these areas with their urban environments, provide them with facilities and high quality public spaces, as well as to encourage local civil community involvement. On the basis of the experiences and tools that have been developed over the past decade in Andalusia, this paper analyzes the integral renovation of the so called San Martin de Porres district. Here we study its urban and architectural development -standing out the work of the architect Rafael de La-Hoz-as well as the social transformations of its population. From the study of the design, management and execution of its renovation the most relevant aspects are deduced with the objective of facilitating their consideration in future renovation policies.

Keywords: Districts; integral renovation; city; social; Córdoba; Rafael de La-Hoz.

(*) Jefa Sección Rehabilitación de Edificios, Agencia de Vivienda y Rehabilitación de Andalucía, Junta de Andalucía. Sevilla (España).

(**) Jefe de Servicio de Rehabilitación de Vivienda, Dirección General de Rehabilitación y Arquitectura, Junta de Andalucía. Sevilla (España).

Persona de contacto/Corresponding author: josei.rodriguez@juntadeandalucia.es

Cómo citar este artículo/Citation: Morón Serna, E., Rodríguez Galadí, J.I. (2015). Rehabilitación integral de barriadas con dificultades sociales en Andalucía. La experiencia de San Martín de Porres en Córdoba. Informes de la Construcción, 67(EXTRA-1): mo27, doi: http://dx.doi.org/10.3989/ic.14.051.

Licencia/License: Salvo indicación contraria, todos los contenidos de la edición electrónica de Informes de la Construcción se distribuyen bajo una licencia de uso y distribución Creative Commons Reconocimiento no Comercial 3.o. España (cc-by-nc). 


\section{INTRODUCCIÓN}

El fin de la burbuja inmobiliaria ha puesto de manifiesto las contradicciones inherentes a los desarrollos urbanos expansivos de la última década. La profunda crisis del sector de la construcción, las insoportables tasas de desempleo, el drama de los desahucios y el excedente de viviendas desocupadas, junto al consumo insostenible del territorio, caracterizan el colapso de un modelo que antepuso el valor de inversión a otras consideraciones. En este contexto, la recuperación del parque residencial existente constituye el principal desafío de las políticas de vivienda. Posibilitar el encuentro entre demanda y viviendas desocupadas, así como la puesta a punto de los parques obsoletos, supone una alternativa sostenible al fracaso del paradigma del continuo crecimiento urbano.

La recuperación de las barriadas construidas en la segunda mitad del siglo xx constituye un reto principal. En primer lugar, por razones cuantitativas, desde la década de los 40 las grandes ciudades andaluzas experimentaron un notable crecimiento poblacional, impulsado por la emigración rural, en ascenso hasta iniciados los 70. Si en un principio las necesidades de vivienda se resolvieron mediante la densificación de las estructuras urbanas y los asentamientos marginales, a partir de mediados de los 50 el Estado, junto a otras instituciones de carácter público y, posteriormente, cuando la rentabilidad estuvo asegurada, la iniciativa privada, afrontaron la promoción de numerosos conjuntos residenciales en las periferias (1). Buena muestra de la escala de esta transformación es que cerca del $40 \%$ de las viviendas principales existentes en Andalucía fueron construidas en el periodo 1951-80 (2). En segundo lugar, por razones sociales, las áreas resultantes de estos crecimientos, alojan en la actualidad a un amplio sector de población afectada por problemas socioeconómicos, a veces en riesgo de exclusión social. Resulta significativo que las zonas desfavorecidas de las principales ciudades andaluzas coincidan, en su mayor parte, con barriadas construidas en la segunda mitad del siglo xx (3).

La degradación que hoy afecta a muchas de estas barriadas tiene su raíz en el mismo modelo de crecimiento que alentó su origen. En ellas, la dimensión y escala, el carácter unitario y la monofuncionalidad perviven junto a la segregación social, el aislamiento urbanístico y los déficits de equipamientos. El deterioro físico y las carencias funcionales, de confort y dotacionales se hacen patentes en unos conjuntos construidos bajo criterios mínimos de habitabilidad, con urgencia y limitaciones económicas, materiales y tecnológicas. Su baja o nula eficiencia energética y las carencias de accesibilidad se evidencian ante una población hoy envejecida y presa de la pobreza energética. La deriva social de algunas barriadas, junto a la administración deficiente de un parque residencial muchas veces de titularidad pública (en gran parte traspasado desde el Estado a la Comunidad Autónoma a partir de 1984 y otras veces aún adscrito a las entidades municipales o patronatos benéficos que las promovieron), han favorecido en ocasiones la ocupación, el traspaso irregular o la utilización para fines no residenciales de viviendas adjudicadas en su origen en alquiler o acceso diferido a la propiedad con largos periodos de amortización, generando inseguridad, dificultando la convivencia vecinal y acelerando su degradación física (4).

La recuperación de estas barriadas requiere, por tanto, actuaciones integrales que al tiempo corrijan el deterioro y las ca- rencias de los edificios, mejoren las relaciones con su entorno urbano y las doten de equipamientos y espacios públicos de calidad, haciendo partícipes a los vecinos y favoreciendo su desarrollo social y económico.

\section{EXPERIENCIAS E INSTRUMENTOS}

El análisis de las políticas de rehabilitación en Andalucía de los últimos 30 años, evidencia que algunas de las consideraciones anteriores fueron ensayadas muy pronto por la administración autonómica. Si las experiencias iniciales alcanzaron mayor intensidad en centros históricos como el de Cádiz (5), interesa aquí destacar otras como la del barrio de La Chanca de Almería, que aún mantenía la situación de «miseria insostenible» denunciada por Juan Goytisolo a comienzos de los 60 (6) y retratada desde unos años antes, con una mezcla de desolación y belleza, en las fotografías de Pérez Siquier (7). En este caso las prioridades fueron establecidas mediante la participación canalizada a través de la asociación de vecinos y el equipo redactor del PERI aprobado en 1990. Desde entonces, se han desarrollado actuaciones públicas de suelo, urbanización, rehabilitación residencial, promoción de viviendas y operaciones de realojo, a través de una oficina de rehabilitación gestionada con la colaboración de los vecinos.

Especial interés representa la experiencia de Molino Nuevo en el Polígono Almanjáyar de Granada, conjunto de 160 viviendas de principios de los 80 cuya gestión había sido desatendida desde un principio por sus promotores, facilitando el rápido deterioro de sus condiciones sociales y de alojamiento. Las actuaciones de rehabilitación, iniciadas al final de los 90 con la colaboración de la Asociación Gitana Anaquerando, se plantearon como un instrumento de intervención social que implicaba la recuperación física de las viviendas junto al desarrollo de iniciativas de participación con los residentes (regulación de la ocupación de las viviendas a través de una oficina habilitada en el barrio y formación de los jóvenes en una escuela taller para su posterior inserción sociolaboral en la rehabilitación) (8).

Las experiencias anteriores y, en particular, las fórmulas de financiación y gestión ensayadas en Cádiz a partir de 1999, sustentaron el diseño de los instrumentos para la rehabilitación integral de ámbitos urbanos que los Planes andaluces de vivienda incorporaron en la última década. Las Áreas de Rehabilitación Concertada (ARC), aunque enunciadas ya en el III Plan Andaluz de Vivienda y Suelo 1999-2002, se articularon con detalle e impulsaron de manera decidida en el IV Plan Andaluz 2003-2007, como instrumento específico de intervención en ámbitos históricos con valor patrimonial. La regulación de las ARC flexibilizó la aplicación de los programas ordinarios de ayudas, posibilitando el diseño de otros específicos para cada ámbito. Se establecieron oficinas técnicas en las zonas de actuación, así como comisiones para la coordinación entre administraciones y la participación de los colectivos vecinales. La falta de sujeción en Cádiz a un programa de objetivos y actuaciones previamente definido se corrigió con la exigencia de redacción de programas de actuación específicos que precisaran los tiempos de ejecución, las inversiones y los compromisos asignados a los distintos agentes.

Pero además, junto a las ARC, el IV Plan estableció la Rehabilitación Integral de Barriadas (RIB) como «instrumento global de intervención sobre ámbitos urbanos constituidos por conjuntos residenciales centrales y periféricos en proceso de 
degradación, afectados por graves problemas habitacionales y de integración social, con el objetivo de mejorar sus condiciones de alojamiento y otros aspectos de carácter urbanístico y social» (9). Aunque su instrumentación, financiación y gestión no difirió sustancialmente de las ARC, su inclusión en el Plan, con el objetivo de identificar de manera diferenciada las actuaciones en áreas residenciales con especiales problemas sociales, permitió impulsar a partir de 2003 la rehabilitación integral de numerosas barriadas andaluzas de la segunda mitad del siglo xx. Destacan por su envergadura las actuaciones de rehabilitación iniciadas en el 2005 en el Polígono Sur en Sevilla, una de las expresiones más dramáticas de la segregación social del espacio urbano en Andalucía, así como las comenzadas en El Puche en Almería (2004), La Piñera y El Saladillo en Algeciras (2007), Polígono Almanjáyar de Granada (2004) o San Martín de Porres en Córdoba (2004).

Entre ellas, las actuaciones en esta última barriada son las únicas próximas a finalizar. Esta circunstancia, junto a sus características históricas, urbanísticas, arquitectónicas y sociales y la dimensión integral de las intervenciones, resalta el interés de esta experiencia, facilitando también una mayor concreción en su análisis y evaluación. Con el objetivo de desvelar sus aspectos más relevantes y con capacidad de referencia para el di- seño de futuras intervenciones, en lo que sigue, se aborda el estudio del origen y desarrollo urbano, arquitectónico y social de San Martín de Porres en el Sector Sur de Córdoba; se analiza el proceso de rehabilitación, considerando tanto aspectos de la concertación, planificación y gestión de las actuaciones, como sus principales características técnicas; para finalizar sintetizando, a modo de conclusiones, los aspectos que, se entiende, tienen una mayor capacidad de transferencia.

\section{SAN MARTÍN DE PORRES Y EL SECTOR SUR DE CÓRDOBA}

La imagen aérea de Córdoba de 1956 (Figura 1) permite observar el contexto previo al fuerte desarrollo expansivo de la década siguiente, donde la construcción del Sector Sur será protagonista. La margen izquierda del Guadalquivir, frente a la ciudad histórica, constata el carácter agrícola y extraurbano que hasta entonces dominó la periferia meridional de la ciudad. Sigue siendo reconocible, en el entorno del arranque sur del puente romano, una exigua trama histórica que «nunca llegó a superar la dimensión de un arrabal extramuros de condición suburbana» (10). En contraste, por tamaño y geometría, destaca un extenso y denso desarrollo residencial de una sola planta. Se trata de una de las primeras respuestas

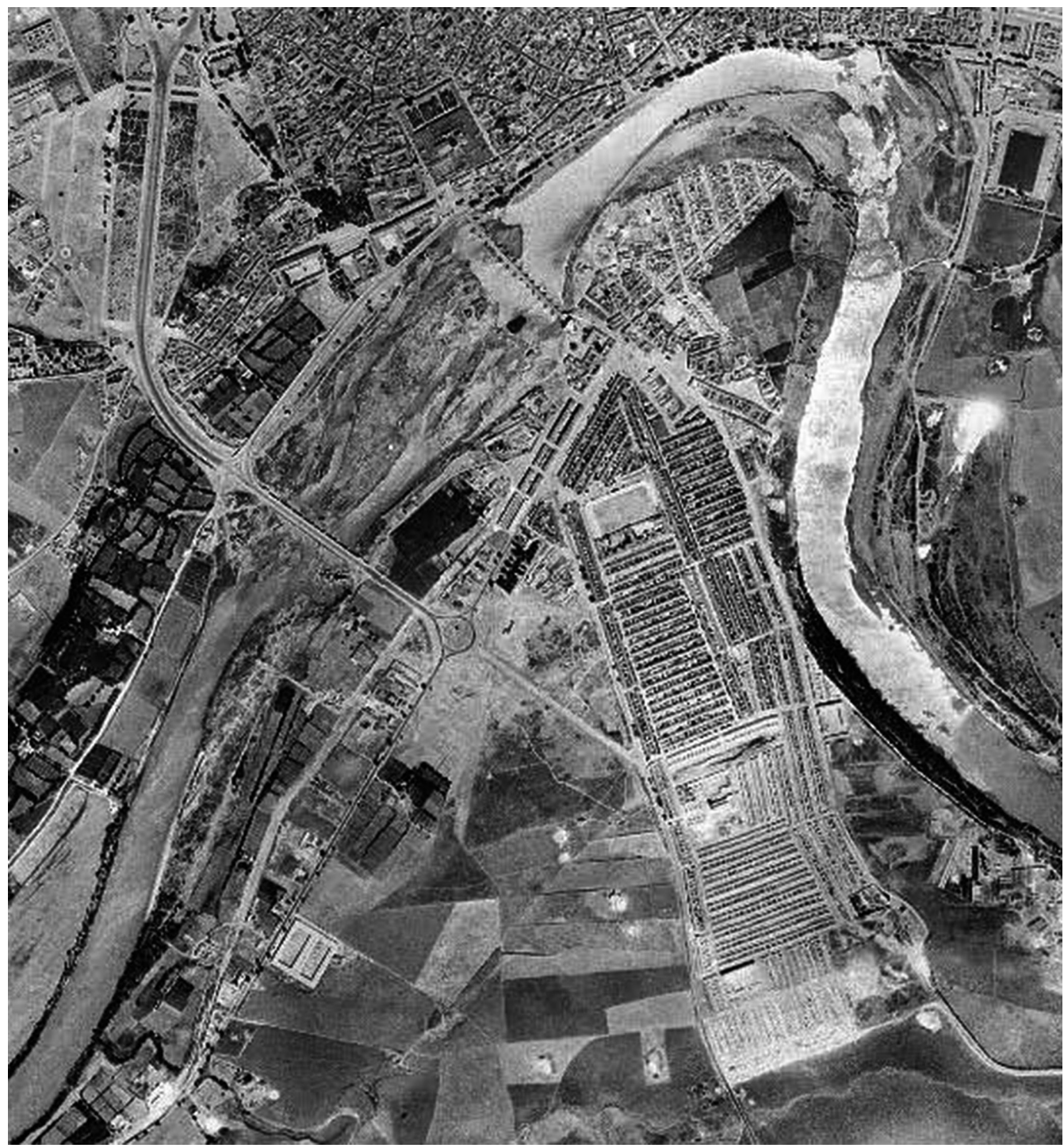

Figura 1. Córdoba. Imagen aérea. «Vuelo americano». 1956. 
al acuciante problema de escasez de vivienda que de manera dramática azotó en aquellos años y en la década siguiente a la población cordobesa con menores recursos. Construida a partir de 1949, por iniciativa del obispo Fray Albino González, esta barriada inauguró el destino residencial de carácter social de esta margen del río, corroborado en sus posteriores desarrollos (11).

Operaciones como la de Fray Albino no alcanzaron sino a mitigar parcialmente el problema. Durante los años 40 y 50, Córdoba experimentó uno de los mayores incrementos de población entre las capitales andaluzas (12), principalmente por la masiva llegada de población rural, en su mayoría de la provincia. La falta de alojamiento pronto se tradujo en la generación de extensos asentamientos de chabolas y chozos en diversos enclaves de la periferia urbana, como el reconocible en la imagen, a espaldas del pequeño arrabal histórico, abrazado por el río y expuesto a sus avenidas.

Pero la imagen también registra otros elementos que remiten al inminente desarrollo urbanístico de este sector. Destaca el Puente de San Rafael, inaugurado en 1953, que generó en ambas orillas los ejes estructurantes de las nuevas expansiones hacia el sur y el oeste. Serán precisamente la prolongación de su directriz hasta el cruce con la carretera de Granada y el trazado en perpendicular, paralelo al cauce, de la carretera de Cádiz, las líneas vertebradoras del crecimiento urbano que, bajo la denominación de «Sector Sur», incorporará en los años siguientes los terrenos más suroccidentales. Su urbanización, cuya ejecución se percibe iniciada en la imagen, fue aprobada en 1955 y desarrollada en los años siguientes tras la adquisición municipal de los terrenos para su posterior venta a diversos promotores.

La urbanización del Sector Sur adelanta las determinaciones del PGOU de $1958^{1}$, que prevé grandes polígonos residenciales colmatando los vacíos intercalados por el crecimiento discontinuo anterior. En la búsqueda de un continuo urbano, la barriada de Fray Albino marcará el límite del nuevo crecimiento y su geometría tendrá reflejo en el trazado de los ejes que confluyen en la plaza del Mediodía, espacio central de la nueva expansión. El Plan estableció inicialmente una zonificación diferenciada: «bloque abierto» en el área más próxima al Guadalquivir e «intensiva baja» para San Martín de Porres. Aunque finalmente el bloque se extenderá a todo el Sector Sur, las características topográficas del terreno, elevado más de 10 metros en San Martín de Porres, determinarán el carácter diferenciado de este barrio y el aislamiento urbanístico que condicionará su evolución.
En su conjunto, la construcción del Sector Sur en los años siguientes, donde destaca la participación del arquitecto Rafael de La-Hoz ${ }^{2}$, constituirá la primera oportunidad en Córdoba para el desarrollo de una experiencia residencial coherente con el debate más avanzado sobre la vivienda social producido en España en los años previos.

La década de los 50 sitúa la necesidad de vivienda y las propuestas del movimiento moderno, en su consecuente debate acerca de las variaciones tipológicas y formales, en tema protagonista para la arquitectura a escala internacional (13). En España, se vive una fase crucial donde se retoman las experiencias anteriores a la guerra civil y la arquitectura triunfalista de la dictadura convive con proyectos tan humildes y limitados en presupuesto como interesantes para la historia de la vivienda de promoción pública (14).

La trayectoria profesional de Rafael de La-Hoz, en sus inicios, se inscribe en la superposición de ambos procesos. En un primer momento su aportación resulta aislada. La recomendación de Cabrero en 1954 no fue suficiente para su inclusión en el concurso de aquel año, tampoco en el de 1956 (15). Pero sus proyectos de viviendas de aquellos años se han convertido en referencia insoslayable, en su carácter de experimentación e innovación, para el estudio de la arquitectura residencial de ese periodo.

Su investigación se desarrolla en dos vertientes, las soluciones constructivas y la organización compositiva, con un único objetivo: «construir una vivienda con la mitad de la superficie universalmente considerada mínima, con la mitad del presupuesto que realmente precisa» (16), reduciendo al mínimo la superficie no habitable para obtener la máxima utilidad del metro edificado y descargar su coste. Establece dos vías: la convertibilidad de usos, con actividades superpuestas, que ha de hacerse sobre elementos ya existentes sin necesidad de añadir mecanismos adicionales, con la única solución de que la vivienda se entregue completamente amueblada, y una segunda vía, de máximo aprovechamiento en la menor superficie, que implica una investigación sobre la organización de la vivienda para acoger el programa 3-6 (para seis personas con tres dormitorios) (Figura 2). Concluye optando por el modelo de agrupación de dormitorios y aseo alrededor de un pequeño distribuidor, que conecta la estancia, disponiendo en ésta la circulación en diagonal que despeja los ángulos para posibilitar sus funciones. Fija las variables óptimas: el número de plantas en cinco (mejor proporción de coste $\mathrm{m}^{2}$ por superficie útil), el de crujías en cuatro $(12,97 \mathrm{~m}$, siguiendo los cálculos de W. Triebel) y la distribución de cuatro viviendas

\footnotetext{
${ }^{1}$ Nacido al amparo de la Ley del Suelo de 1956, fue redactado por el arquitecto José Rebollo Dicenta, también autor del proyecto de urbanización del Sector Sur. Por primera vez se dota a Córdoba de un proyecto global de desarrollo con el objetivo de articular el crecimiento y las áreas de expansión.

${ }^{2}$ Rafael de La-Hoz Arderius (1924-2000), arquitecto titulado por la Escuela Técnica Superior de Arquitectura de Madrid en 1951, amplió sus estudios en el Massachussets Institute of Technology en 1955. Inició su actividad en Córdoba, donde mantendría estudio profesional hasta mediados de la década de los 70, cuando se traslada a Madrid. Sobresalen entre su primeras obras, las tiendas y chalés proyectados en la ciudad de Córdoba, la sede de la Cámara de Comercio de Córdoba (1953) y el Colegio Mayor Aquinas en Madrid (1953, Premio Nacional de Arquitectura en 1956), éstas últimas obras en colaboración con José María García de Paredes. Sus primeros trabajos evidencian ya la apuesta por la renovación creativa y la innovación tecnológica que estarán presentes a lo largo de su producción arquitectónica pero que tuvieron especial trascendencia en contraste con el cerrado contexto político y cultural de sus inicios. Interesa destacar aquí su activo papel en la investigación y producción de la vivienda social en los años a caballo entre las décadas de los 50 y 6o, del que constituyen buena muestra sus proyectos en el Sector Sur de Córdoba. Compaginó la actividad profesional con el desempeño de responsabilidades institucionales (entre otras, fue titular de la Dirección General de Arquitectura y Tecnología, desde donde promovió en 1971 la redacción de las Normas Tecnológicas de la Edificación). En 2000, a título póstumo, le fue concedida la Medalla de Oro de la Arquitectura.
} 


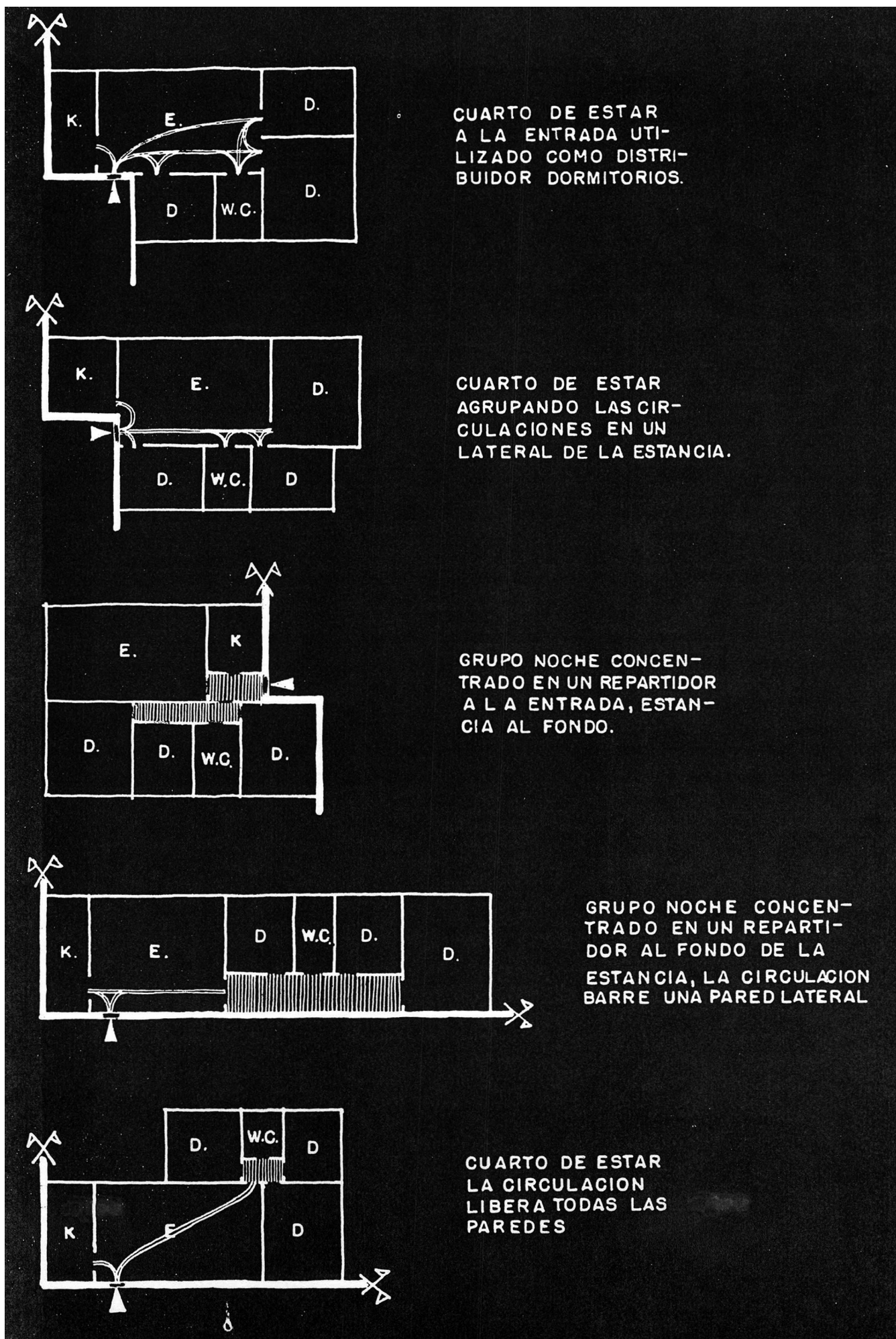

Figura 2. Variables de composición 3-6. Rafael de La-Hoz Arderius. 1962.

por planta a un solo nivel. Y desde el análisis de ejemplos conocidos del programa 3-6, considerados en sus soluciones de escalera, define su propuesta de cocina lineal, que simplifica instalaciones y trabajo, pequeña terraza lavadero, y escalera iluminada a fachada.

El indudable interés de la investigación se materializa en una serie de realizaciones que exploran ambas vertientes. Entre ellas, es preciso destacar las viviendas ultrabaratas en Palma del Río (1951), superficie mínima de $20 \mathrm{~m}^{2}$, agrupación en cuatro, con un sistema estructural de catenaria invertida (17), el proyecto de viviendas en Montilla (1957), todavía conservando la disposición de dos viviendas por planta y el barrido lateral de la estancia que luego desecharía, y la prefabricación de alojamientos de emergencia en Córdoba (1964) (18), dentro de su apuesta por la industrialización (19).

Sin duda, el Sector Sur de Córdoba supone para Rafael de La-Hoz el ámbito preciso para la experimentación de sus modelos de viviendas económicas, encontrando su espíritu in- 
vestigador el contexto favorable de la tecnocracia franquista de estos años. El resultado pone en evidencia el choque con la dura realidad del país, la carencia de recursos, la urgencia e inmediatez de las soluciones y la precariedad de infraestructuras e insuficiencia técnica (20).

En los terrenos más suroccidentales la construcción de viviendas se había iniciado en 1957, con los primeros grupos promovidos por La Obra Social Cordobesa de Huertos Familiares $^{3}$ (proyecto de Romero Rivera) y el Ayuntamiento de Córdoba (Rebollo Dicenta). Les siguieron dos promociones de la Obra Sindical del Hogar (OSH) (De La-Hoz y Escribano Ucelay, respectivamente) y algunas promotoras privadas (De La-Hoz, entre otros). Los terrenos restantes, entre las avenidas de Cádiz y Granada, se ocuparon a principios de los 60, por iniciativa municipal de nuevo, junto a cooperativas y cajas de ahorro (principalmente De La-Hoz). Hacía el final de esa década, se culminó el proceso con inmobiliarias privadas en los últimos solares alineados con la avenida de Granada.

De las más de 4.800 viviendas construidas hasta mediados de los 60 (Figura 3) -conjunto representativo del debate en

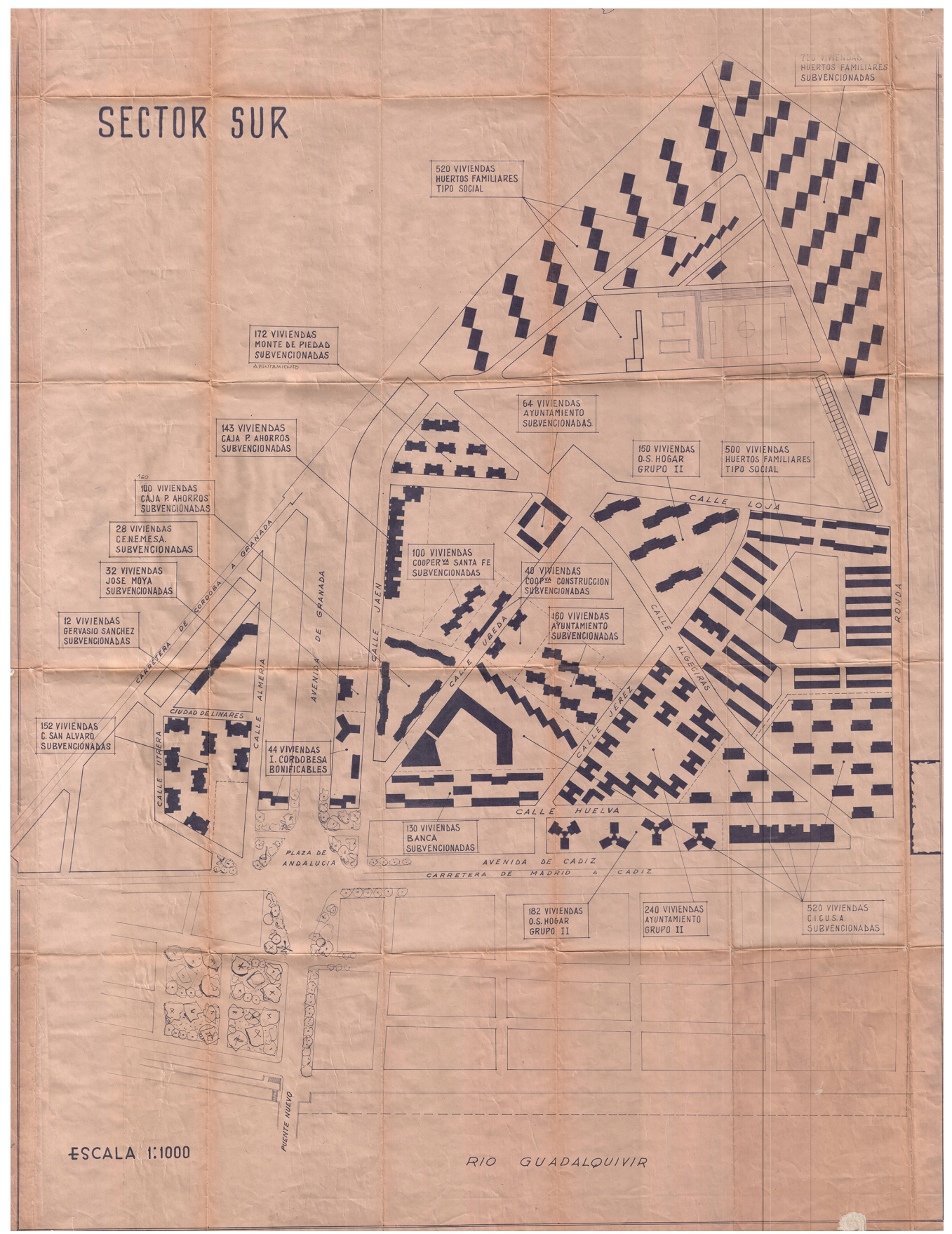

Figura 3. Sector Sur. Rafael de La-Hoz. 1962.

\footnotetext{
${ }^{3}$ La Obra Social Cordobesa de Huertos Familiares fue constituida en 1952 a instancias del Gobierno Civil, como organismo intermediario entre el Instituto Nacional de Colonización, municipios y huertanos, dotándola de funciones de administración, coordinación, promoción y asesoramiento en el establecimiento de huertos familiares. A finales de 1953 fue declarada «entidad benéfica constructora», acogida al régimen de protección de vivienda.
} 
torno a la vivienda social, su arquitectura y su promoción-, la mayoría fueron calificadas como «subvencionadas», régimen de protección que en 1957 vino a diluir el protagonismo público en favor del sector privado, iniciándose una etapa de política especulativa.

San Martín de Porres tuvo su deriva particular. En 1960, Huertos Familiares comenzó la construcción de 520 viviendas junto a la carretera de Granada (sector calle Torremolinos). Dos años después, la misma promotora inició 720 viviendas más (sector calle Motril) y cedió en venta a la $\mathrm{OSH}$ el terreno sobrante para otras 700 viviendas (grupo Los Dolores). Las 1.940 viviendas resultantes, agrupadas en 97 bloques de similares características, estaban concluidas a finales de 1965, según proyecto de Rafael de La-Hoz.

La aportación de Rafael de La-Hoz en el Sector Sur -con más de 3.400 viviendas, entre públicas y privadas, sin duda el arquitecto con mayor presencia- supone avanzar en la optimización de los conceptos de vivienda económica y su agrupación. Partiendo del bloque de dos viviendas por escalera y planta, ya ensayado en Montilla en 1957, desarrolla diversas propuestas de bloque compacto de cuatro viviendas por planta. La disposición de los bloques, en diversas tipologías de acoplamiento, destacando la versión de unión en espiga con respecto a una calle central utilizada en San Martín de Porres, refuerza la compacidad, subrayada por la propia eliminación de los patios interiores (21). Pese a la austeridad de los materiales empleados (hormigón y ladrillo visto), no se descuida el aspecto exterior, con cierto dinamismo por la creación de zonas en sombra a partir de los rehundimientos en fachada. La imagen exterior alimenta la ambivalencia de escasez y rigor: cubiertas a dos aguas, planos de ladrillo visto solo matizados por las bandas ho- rizontales de los forjados y las secuencias verticales de la distribución de los huecos.

Pero al abordar el origen urbano de una barriada social como la de San Martin de Porres, no es fácil escapar de los tópicos y reparar en que detrás de las expresiones habituales, repetidas, hay personas y tiempo. Se escribe una y otra vez «oleadas de emigración», "necesidades de vivienda», «expectativas de una vida mejor», «huida del hambre»... Y todo parece pertenecer a un pasado ajeno, distante, que se ha quedado en las hemerotecas de las fechas indefinidas. Ha quedado dibujado el cliché visual de una realidad que es preciso restituir a nuestra propia historia (Figura 4). Las chabolas y los chozos, la amenaza de las crecidas del río y la vulnerabilidad ante las inundaciones, los alojamientos provisionales... no son palabras de Rafael de La-Hoz, del Gobernador Civil o del obispo de la diócesis, son nuestra biografía como sociedad.

El relato de la miseria que se traslada del campo a la ciudad es también la deriva histórica de la autarquía a la tecnocracia. Desde la perspectiva económica, no es sino la concurrencia de dos factores básicos para el desarrollo: la inversión del capital, atento al máximo beneficio del corto plazo, junto a la afluencia de una gran masa de mano de obra barata en precarias condiciones de existencia (22). Ciertamente, se puede considerar así mismo como un fenómeno con grave repercusión en la estabilidad social y en el orden político. De ahí las distintas formas de caridad y la iniciativa de las instituciones eclesiásticas, o la propia respuesta del Estado con la promulgación del Plan Nacional y la Primera Ley del Suelo, y la posterior creación del Ministerio de la Vivienda, todo a la espera de un capital privado que aún no se interesa por una emigración a la que no considera negocio dotar de vivienda por tratarse de una demanda no solvente.

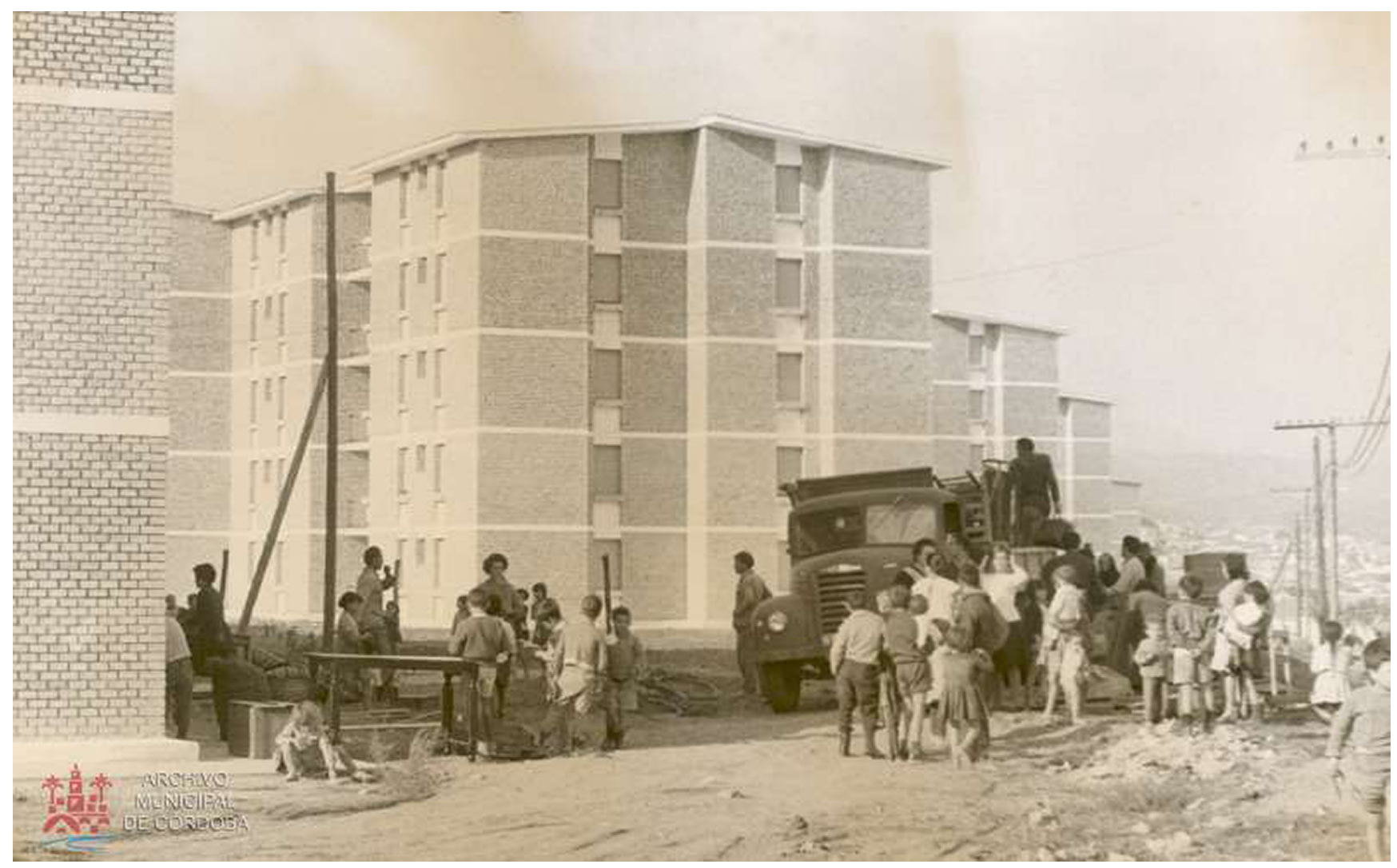

Figura 4. Escena de mudanza. Sector calle Torremolinos. Archivo Municipal de Córdoba. Autor desconocido. 1965. 
Las transformaciones nos ofrecen una visión divergente de la imagen fija que esperábamos. La experiencia agraria llevada a cabo por el Instituto Nacional de Colonización desde 1950 se desnaturaliza con una extraordinaria rapidez. Si en poco más de un año el número de proyectos acogidos a la iniciativa de Huertos Familiares se aproximó a 1.400, la mayoría fueron abandonados en los años siguientes. De las modalidades previstas como mejoras para la puesta en el mercado de la tierra (parcelación, instalación de regadío y construcción de viviendas donde fuera necesario) apenas quedó la promoción de viviendas, operando como una promotora social de trayectoria efímera. De modo similar, el cumplimiento de los planes de vivienda de la $\mathrm{OSH}$, apenas se reconoce en la euforia de la prensa servil de la época que repetía la voluntad del gobierno de construir en diez años 550.000 viviendas de renta limitada. Si en las condiciones normativas la situación del demandante se presentaba más que favorable (créditos del Instituto Nacional de Vivienda (INV) sin interés por el 80\% del precio de venta a cincuenta años), finalmente apenas se construyeron la mitad de las viviendas previstas y la mayoría de ellas correspondientes a viviendas de categoría superior, financiadas con los fondos de las de tipo social (15).

Tras las cifras, los módulos y las cuotas, el cambio profundo de personas y familias cuyas vidas se transforman de raíz en sus parámetros más esenciales. El espacio constreñido, los límites subrayados, el tiempo en manos ajenas y la sensación lacerante del desarraigo. Con el abandono y la perpetuación de la marginación como únicos horizontes, son estas personas quienes van a hacer cola a la puerta del Dispensario de Carlos Castilla del Pino, testigo analítico de esta cara desencajada de la ciudad que crece (23).

\section{REHABILITACIÓN DE SAN MARTÍN DE PORRES}

Al final del pasado siglo conviven procesos difícilmente reconciliables en torno a la deriva de la barriada de San Martín de Porres. La administración autonómica, responsable de las competencias de vivienda, se ve atrapada en el debate entre sustitución y rehabilitación para unas viviendas de valoración creciente en el ámbito arquitectónico, pero también de deterioro imparable y objeto de una importante reivindicación social. La decisión de incluir las viviendas que conforman esta barriada en el catálogo del movimiento moderno de Andalucía contrasta con la ausencia de una estrategia global de actuación. Será precisamente la primera demolición, acometida por el Ayuntamiento en 1999 sobre uno de los bloques más degradados de la calle Torremolinos, sector con mayores problemas sociales, la que desencadene como necesario e irreversible el proceso de concertación, única salida viable para la barriada.

Si el PGOU de 1986 pasó por alto el proceso de degradación ya iniciado en San Martín de Porres, el de 2001 no cerró la solución a adoptar. La voluntad política de acuerdo para la intervención entre las administraciones autonómica y municipal adquiere forma definida en la redacción de un Programa de Actuación en $2003^{4}$ (Figura 5) y la posterior declaración de San Martín de Porres como barriada de rehabilitación integral (RIB) en 2004, en el marco del IV Plan Andaluz de Vivienda y Suelo. Se delimita el ámbito de actuación y se establecen los diferentes órganos interadministrativos de coordinación, seguimiento y participación ciudadana, con una oficina técnica en contacto directo con las asociaciones y la población residente.

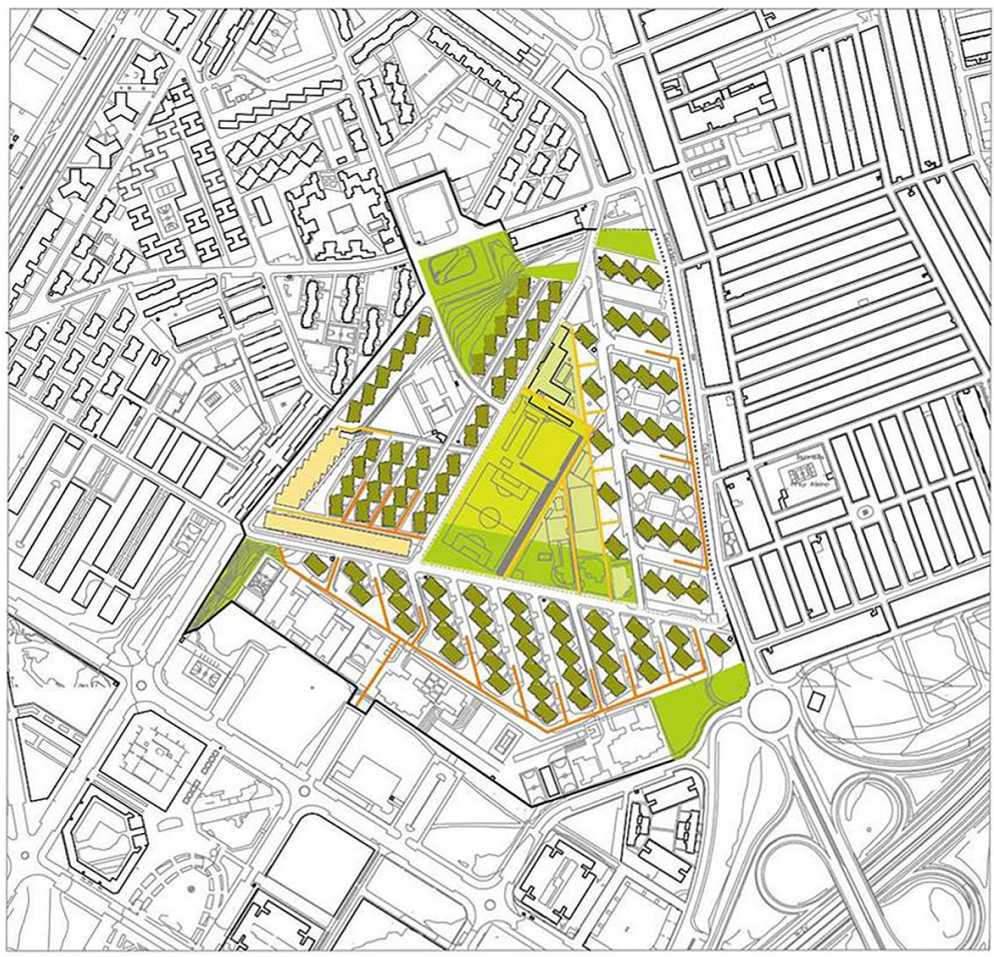

PROGRAMA DE TRANSFORMACIÓN URBANA Y EDIFICATORIA SS-7

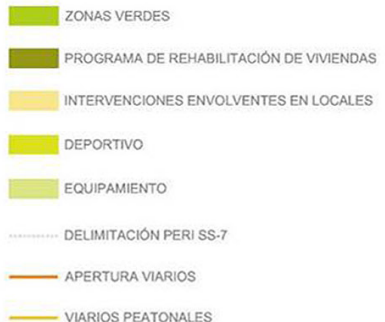

Figura 5. Delimitación y esquema de actuaciones. Programa de Actuación San Martín de Porres. 2003.

\footnotetext{
4 Redactado por los arquitectos José Canovaca y Rafael Suárez, el Programa de Actuación para la Rehabilitación Integral de la barriada de San Martín de Porres fue aprobado por el Pleno del Ayuntamiento de Córdoba en mayo de 2003 y por las Direcciones Generales de Arquitectura y Vivienda y de Urbanismo de la Consejería de Obras Públicas y Transportes en mayo de 2004.
} 
Entre los objetivos y compromisos del Programa de Actuación se incluyeron una serie de intervenciones urbanas que prolongaban la reurbanización y cualificación del espacio público ya iniciadas por el Ayuntamiento. Sobre una trama apenas dibujada, se pretende la diferenciación de los espacios particulares respecto de los públicos (apertura de nuevos viales y cerramiento de espacios exteriores en torno a los bloques) y la generación de topografías, zonas verdes y áreas de encuentro (remodelación de la Plaza del Mediodía, ente otras). Estas actuaciones serían gestionadas y financiadas por el Ayuntamiento. En relación a las viviendas, asumida la necesidad de intervención en su interior para la restitución de unas condiciones mínimas de habitabilidad, el Programa de Actuación propuso incentivar la iniciativa de los vecinos mediante el establecimiento de líneas de ayudas, reservando la intervención pública para las actuaciones de dimensión colectiva, como la adecuación de los elementos y servicios comunes de los edificios. Estas actuaciones serían gestionadas y financiadas por la administración autonómica.

El análisis evidenció que el tiempo se había ensañado con la fragilidad de los sistemas empleados, el abandono en el mantenimiento, las disputas sobre la propiedad y las ampliaciones a costa del espacio público. Lo más visible, las transfor- maciones: la incorporación de la terraza-lavadero, cerrada a la cocina; la ampliación del salón con la supresión de un dormitorio; los cambios en la carpintería exterior, modificada con ventanas de aluminio anodizado... Pero en el diagnóstico y en las prioridades de la intervención pesan otras cuestiones más esenciales. Una circunstancia muy favorable fue el buen estado estructural: un sistema de cimentación singular y adecuado al suelo expansivo, pozos de hormigón atados por una losa armada continua en toda la planta baja, cerramientos exteriores con muros de carga de un pie de ladrillo visto, crujías interiores con muros de carga (calle Torremolinos) o con estructura de pilares (calle Motril y Los Dolores), forjados de $15 \mathrm{~cm}$ de espesor de viguetas unidireccionales in situ con bovedillas cerámicas.

Iniciado el proyecto de intervención, se concluyó que en la vertiente constructiva radicaba también el valor patrimonial de estos conjuntos de viviendas representativos de la más cualificada arquitectura de la vivienda social de principios de los 60 en Andalucía. De ahí que finalmente se opte por conservar los muros de ladrillo visto. De acuerdo con el informe inicial del arquitecto Jorge Benítez, quien lidera y coordina, al frente de la oficina de rehabilitación, la regeneración del barrio $^{5}$, se desestima la solución de aislar exteriormente los
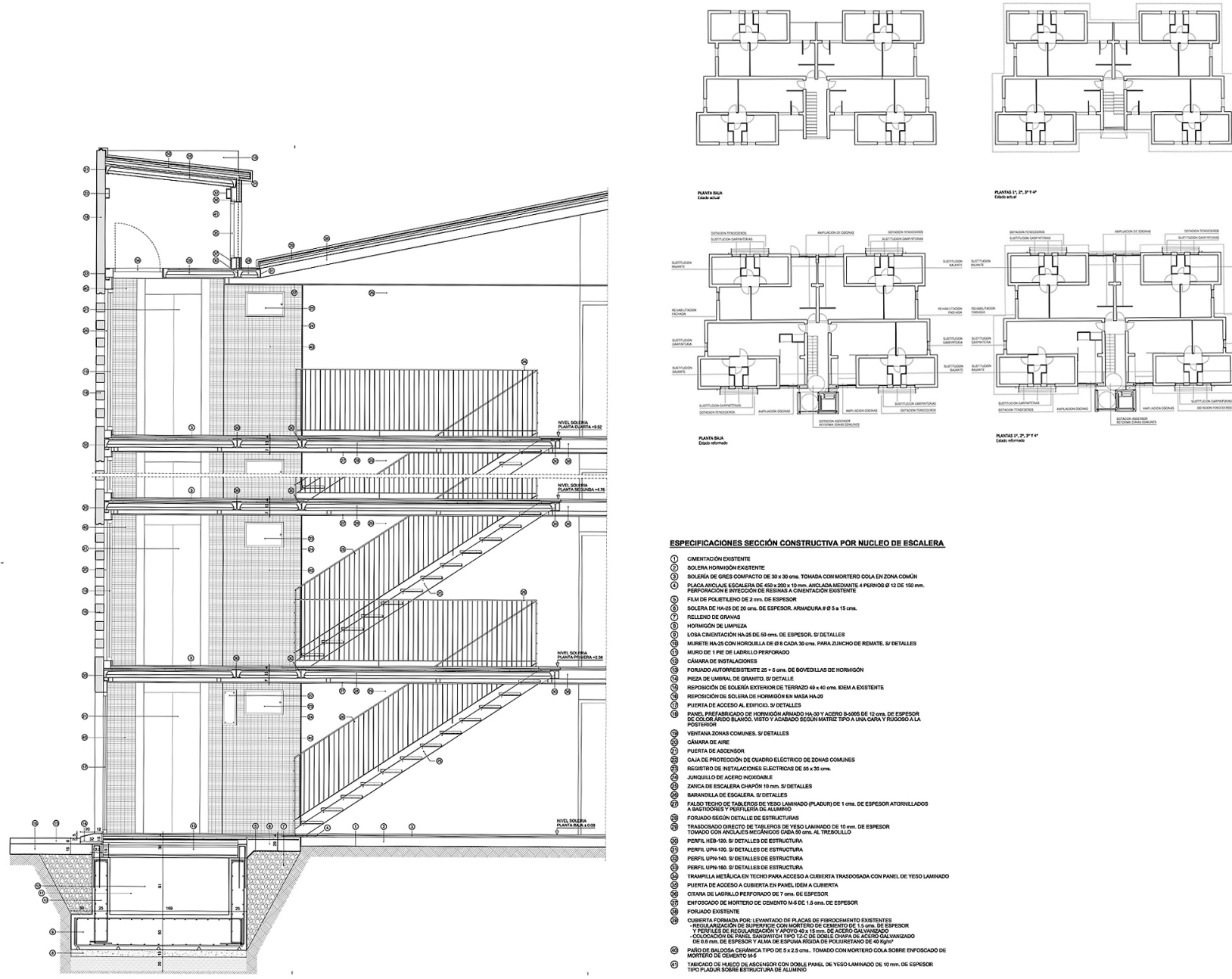

2asmans
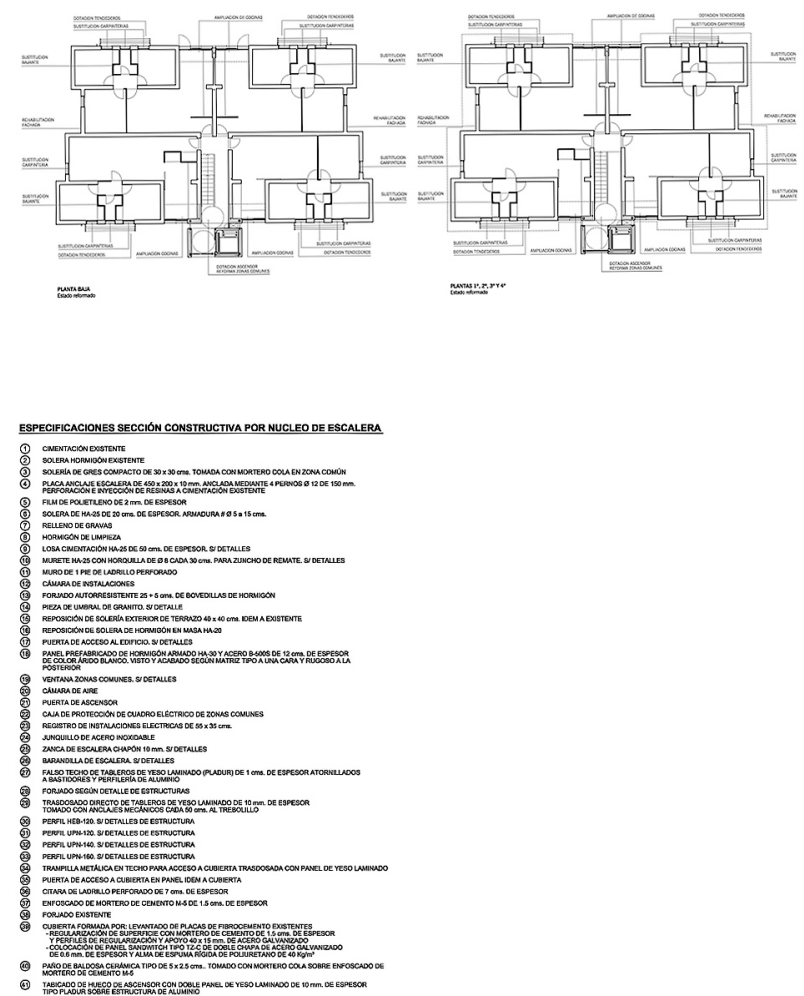

Figura 6. Proyecto de Rehabilitación San Martín de Porres. 2005.

\footnotetext{
${ }^{5}$ Los proyectos de intervención serán posteriormente redactados por los arquitectos Antonio y Pedro Peña, para las zonas de la calle Motril y Los Dolores, y Gabriel Rebollo y Sebastián Herrero para la calle Torremolinos.
} 
edificios y su posterior enfoscado. Las humedades de condensación, descritas en el Programa de Actuación, considera que no poseen relevancia en el conjunto y pueden ser combatidas de forma eficaz aumentando la superficie de ventilación, mientras que un buen acristalamiento sería suficiente para conseguir una mejora equivalente de aislamiento térmico. La decisión es trascendental para decantar una apuesta diferente en el concepto de rehabilitación. Frente a la idea de crear una nueva envolvente para presentar una imagen de actualización, la convicción de que será la propia renovación participada y compatible con los valores de la arquitectura heredada la que propicie la deseada transformación.

No obstante, la intervención necesita eliminar y reorganizar elementos (antenas, tendederos, rejas, climatizadores) que esconden la limpieza compositiva de la fachada, de igual modo, el deterioro de las capas de pintura en las plantas bajas conduce a plantear la solución de pintar toda la fábrica de ladrillo, medida estética y técnica al tiempo, pues redundará en la mejoría de su impermeabilización. Con ese mismo doble valor, se adopta la solución de rasgar los huecos de las ventanas de los dormitorios hasta el suelo. En este caso las ventajas son aumentar la superficie de iluminación y ventilación, especialmente importante para el dormitorio integrado en el estar, resolver las humedades de los antepechos, con medio pie de fábrica de ladrillo, y mejorar a la vez la composición del alzado y las condiciones térmicas y de seguridad con ventanas de acristalamiento doble y persianas de aluminio extrusionado, evitando la rejería.

La mejora de la accesibilidad mediante la instalación de ascensor era un objetivo principal del Programa de Actuación. Para no afectar a áreas privativas, se opta por situarlo, junto a un nuevo núcleo de escaleras, en el exterior de la edificación. Esta solución implica necesariamente cambiar la escalera por una de un solo tramo entre plantas, una remodelación complicada de ejecutar minimizando las molestias de los residentes, que no fueron realojados. La solución proyectada, con una estructura mixta de perfiles metálicos y prefabricados de hormigón texturizado, garantizó la rapidez en la ejecución y una adecuada conexión a la estructura preexistente (Figura 6). Mientras, una escalera provisional desmontable posibilitó el acceso de los residentes.

En las intervenciones de rehabilitación es preciso articular los diferentes objetivos de mejora. En este caso, la ocupación incontrolada del exterior del edificio, en el retranqueo de la zona de los lavaderos, era un problema que debía resolverse junto con la ampliación de las cocinas. La ejecución del núcleo del ascensor hubiera dejado a éstas, adelantadas a la alineación de fachada en las plantas bajas, y en muchos casos de primera y segunda, con deficientes condiciones de iluminación. Se pacta finalmente con los vecinos que el adosado a la edificación no incidirá en los elementos existentes de carpinterías y cerrajerías, ejecutando un forjado, que se dejará en bruto en el interior de las viviendas, y colocando como cerramiento una placa de hormigón texturizado, dotada de las perforaciones que precisa la evacuación de gases. Se consigue así la continuidad con la imagen del nuevo volumen del ascensor, cerrada la intervención con la disposición de una carpintería homogénea en la totalidad del frente y el diseño de una celosía graduable en aluminio.

Considerado en términos de eficiencia energética, el elemento más débil de la arquitectura residencial de estos años suele ser la cubierta, algo que hace totalmente necesaria su sustitución. La existente - placas de fibrocemento ondulado sobre un forjado más, a dos aguas, con pendientes del $14 \%-$ es reemplazada por otra de nueva ejecución, formada por paneles sandwich de acero galvanizado prelacado, que incluyen el correspondiente aislamiento térmico. Esta solución se adapta bien a las pendientes del forjado y a su limitada capacidad resistente.

\section{ASPECTOS RELEVANTES Y CONCLUSIONES}

Cuando finalicen las obras en los últimos 6 bloques de San Martín de Porres (Figura 7) habrán transcurrido 15 años desde la demolición del $n^{0} 35$ de la calle Torremolinos. Dilatado espacio de tiempo para una barriada levantada en un lustro y próxima a cumplir medio siglo. Sin embargo, no ha habido interrupciones señaladas ni incidencias extraordinarias. Cuando se redujo la disponibilidad de fondos autonómicos, se convino en 2009 la cofinanciación con el Estado posibilitando la continuidad de las actuaciones. Los plazos han venido determinados por otras razones: la delicada concertación entre administraciones, los acuerdos con los vecinos, consensuados bloque a bloque, y la especificidad de las viviendas de calle Torremolinos, titularidad de Huertos Familiares en régimen de acceso diferido a la propiedad, pendientes de amortizar y abandonadas en su gestión y administración desde hacía décadas (24). Conviene considerar los tiempos de la rehabilitación de barrios con mayor amplitud que los plazos de ejecución material de las actuaciones, requiriendo previos y duraderos consensos institucionales y con los vecinos.

La actuación estuvo precedida por la acción reivindicativa del movimiento ciudadano, articulado a través de las asociaciones de vecinos. La necesidad de respuesta desde las administraciones determinó la cooperación entre las mismas. Aunque no exento de dificultades, el reparto concertado de responsabilidades en la gestión y financiación de las actuaciones, ha permitido la coordinación de esfuerzos y la integración de acciones urbanísticas, residenciales y sociales. El movimiento ciudadano articulado y la cooperación institucional se revelan imprescindibles para el desarrollo de procesos de rehabilitación integral.

San Martín de Porres, por razones históricas, topográficas, urbanísticas y sociales, partía de una situación de aislamiento que determinó su mayor degradación. Su dimensión y homogeneidad, sin pasar por alto la diversidad social y legal en relación a la ocupación de las viviendas, sustentaron la delimitación del ámbito de actuación y la concreción de los objetivos. La delimitación precisa y dimensión adecuada son factores determinantes para el éxito de las actuaciones: en ámbitos extensos pierden capacidad transformadora, en los reducidos difícilmente alcanzan a incidir en las causas de la segregación.

A diferencia de lo ocurrido en otras experiencias más ambiciosas pero de menor concreción, disponer de un esquema nítido de objetivos y actuaciones hizo posible en San Martín de Porres la evaluación en todo momento de los avances y aplazamientos. Además, la adecuación de los instrumentos a la singularidad de los problemas mediante el diseño de programas específicos de ayudas, concretados en el Programa de Actuación (25) y en la regulación de la participación de la administración autonómica (26), junto al adelanto de las actuaciones de reurbanización al inicio del proceso, facilita- 


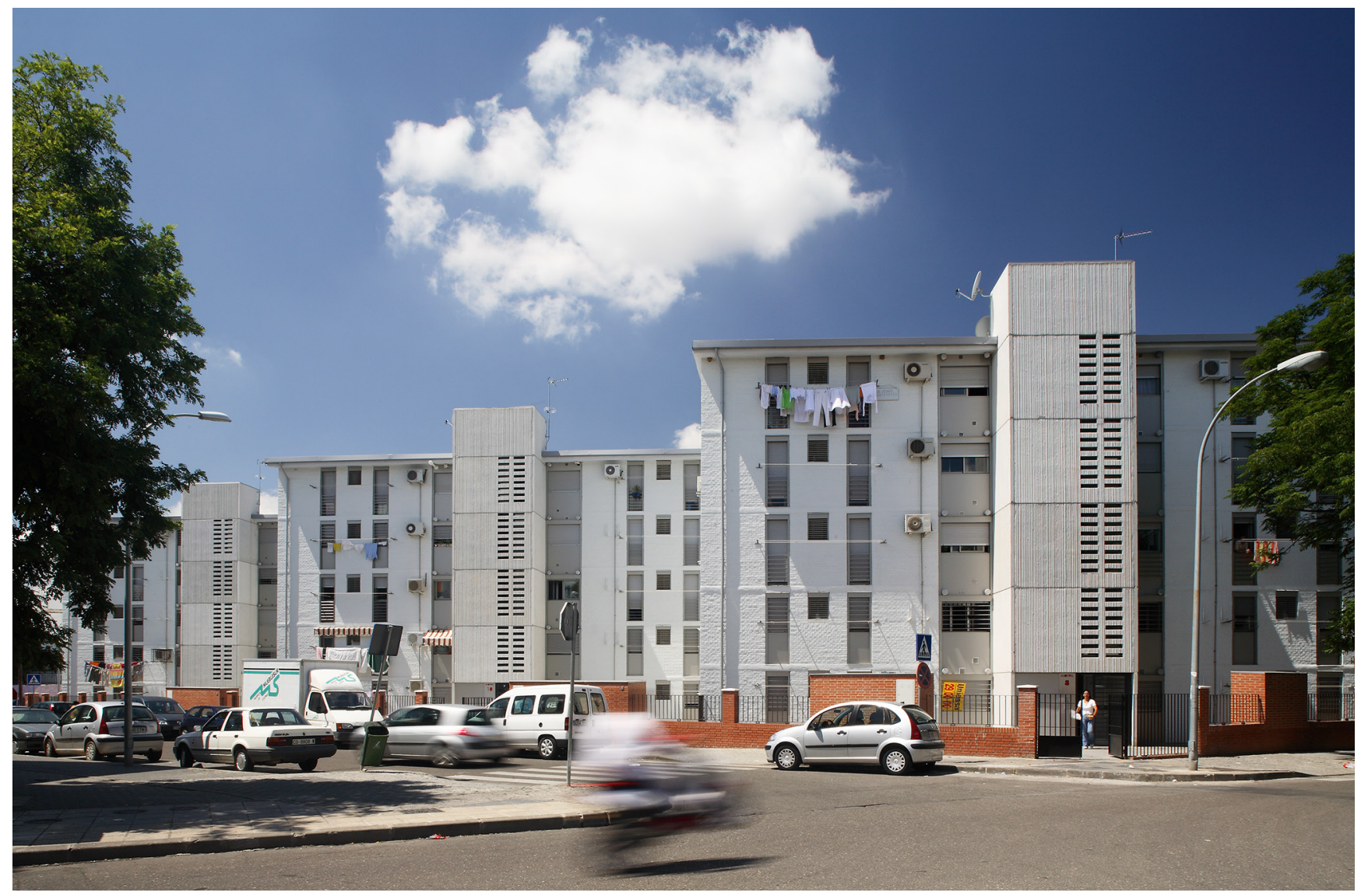

Figura 7. Edificios rehabilitados en San Martín de Porres. Fotografía Manu Trillo. 2007.

ron los acuerdos posteriores con una población escéptica tras 35 años de abandono e incumplimientos. Una programación concreta y, a la vez, flexible de las actuaciones facilitará la adecuación a los problemas que solo la ejecución desvelará.

Por último, destacar la singularidad del caso estudiado, donde se ha compatibilizado la mejora sustancial de las condiciones de alojamiento con la puesta en valor de una arquitectura residencial resuelta en claves de rigor y experimentación a principios de los 60. La profundización en el conocimiento de los barrios de la segunda mitad del siglo xx, avanzando en el estudio de los procesos sociales y la búsqueda de las componentes de identidad de cada uno de ellos, son bases necesarias sobre las que fundamentar la intervención pública en los procesos de rehabilitación de ámbitos estigmatizados durante décadas

\section{REFERENCIAS}

(1) Instituto de Estadística y Cartografía de Andalucía. (2000). Atlas de Andalucía. Tomo IV: Cartografía urbana, Sevilla: Consejería de Obras Públicas y Transportes, Junta de Andalucía.

(2) Dirección General de Arquitectura, Vivienda y Suelo, Ministerio de Fomento. (2014). Observatorio de Vivienda y Suelo. Boletín Especial Censo 2011 Parque edificatorio, Madrid: Centro de Publicaciones, Secretaría General Técnica, Ministerio de Fomento.

(3) Egea Jiménez, C., Nieta Calmaestra, J.A., Domínguez Clemente, J., González Rego, R.A. (20o8). Vulnerabilidad del tejido social de los barrios desfavorecidos de Andalucía. Análisis y potencialidades, Sevilla: Centro de Estudios Andaluces, Consejería de la Presidencia, Junta de Andalucía.

(4) García Vázquez, C. (2010). Obsolescencia urbana: El caso de las barriadas residenciales. La Ciudad Viva, 3: 5.

(5) AVS. (2008). Revitalización urbana. Buenas prácticas, Madrid: Asociación Española de Promotores Públicos de Vivienda y Suelo.

(6) Goytisolo, J. (2001). La Chanca, Sevilla: Consejería de Obras Públicas y Transportes, Junta de Andalucía.

(7) Pérez Siquier, C. (2001). La Chanca, Sevilla: Consejería de Obras Públicas y Transportes, Junta de Andalucía.

(8) Sánchez Comás, F. (2003). Almanjáyar: Construyendo entornos habitables, Sevilla: Ayuntamiento de Granada y Consejería de Obras Públicas y Transportes de la Junta de Andalucía.

(9) Junta de Andalucía. (2005). Artículo 87 del Texto integrado del Decreto 149/2003, de 10 de junio, por el que se aprueba el Plan Andaluz de Vivienda y Suelo 2003-2007, y se regulan las actuaciones contempladas en el mismo, publicado por Orden de 8 de agosto de 2005, Boletín Oficial de la Junta de Andalucía, nº 165, 24 de agosto de 2005, Página 19.

(10) Daroca, F. (2011). El Sector Sur en el planeamiento general de Córdoba. La difícil conquista urbana de La Campiña. En Barrionuevo Ferrer, A. (Ed.), Estudio para la rehabilitación del Sector Sur de Córdoba (pp.227-23o). Córdoba: Empresa Pública de Viviendas Municipales de Córdoba. 
(11) Yllescas Ortiz, M. (2011). Apuntes sobre la situación social y urbanística de la ciudad de Córdoba en los años 50. En Barrionuevo Ferrer, A. (Ed.), Estudio para la rehabilitación del Sector Sur de Córdoba (pp. 215-222). Córdoba: Empresa Pública de Viviendas Municipales de Córdoba.

(12) López Ontiveros, A. (1981). Evolución urbana de Córdoba y de los pueblos campiñeses, Córdoba: Diputación Provincial de Córdoba.

(13) Pérez Escolano, V. (2005). Rafael de La-Hoz Arderius, el arquitecto español contemporáneo. En Rafael de La-Hoz Arderius (pp. 6-15). Córdoba: Fundación Arquitectura Contemporánea.

(14) Sambricio, C. (2008). Vivienda social en tiempos de crisis: sobre las varias crisis vividas en el pasado reciente. En Sambricio, C., Sánchez Lampreave, R. (Eds.), 100 años de historia de la intervención pública en la vivienda y la ciudad (pp. 19-24). Madrid: Asociación Española de Promotores Públicos de Vivienda y Suelo.

(15) Sambricio, C. (2000). De la arquitectura del nuevo estado al origen de nuestra contemporaneidad: el debate sobre la vivienda en la década de los cincuenta. $R A$. Revista de arquitectura, 4: 75-90.

(16) De La-Hoz, R. (1962). La vivienda social. Arquitectura, 39.

(17) Rabasco, P. (2011). El sistema Ctesiphonte. Evolución de la estructura catenaria. Informes de la Construcción, 63(522): 43-52, doi: http://dx.doi.org/10.3989/ic.10.009.

(18) Rabasco, P., López Jiménez, C. (2002, 14-15 de marzo). Córdoba negando a Córdoba. La tensión de la acogida en dos ejemplos de Rafael De La-Hoz. En Congreso internacional Arquitectura, ciudad e ideología antiurbana (pp. 169-177). Pamplona: T6 Ediciones.

(19) De La-Hoz, R. (1960). Plan de Industrialización de construcción de viviendas. Arquitectura, 18.

(20) García Vázquez, C. (1999). Viviendas Fundación Benéfico-Social. Córdoba. Rafael de La Hoz Arderius. En García Vázquez, C., Pico Valimaña, R. (Comisarios), Momo Andalucía. Arquitectura del Movimiento Moderno en Andalucía 19251965 (pp. 238-239). Sevilla: Consejería de Obras Públicas y Transportes, Junta de Andalucía.

(21) Daroca, F. (1991). Sobre la obra de Rafael de La-Hoz Arderius, arquitecto. En Peña Amaro, A., Díaz López, J., Daroca Bruñoa, F., Rafael de La-Hoz. Arquitecto (pp. 9-13), Córdoba: Demarcación de Córdoba del Colegio Oficial de Arquitectos de Andalucía Occidental.

(22) Benítez, J; Asensio, J.M. (1999). Inmigraciones 1950-1965: periferias, polígonos y viviendas sociales. En García Vázquez, C., Pico Valimaña, R. (Comisarios), Momo Andalucía. Arquitectura del Movimiento Moderno en Andalucía 1925-1965 (pp. 208-209). Sevilla: Consejería de Obras Públicas y Transportes, Junta de Andalucía.

(23) Castilla del Pino, C. (2004). Casa del olivo, Barcelona: Tusquets.

(24) Benítez, J. (2008). Experiencia en barrios: San Martín de Porres, Córdoba. La Ciudad Viva, 1: 46-53.

(25) AA.VV. (2003). Programa de Actuación para la declaración del Área de Rehabilitación Concertada «San Martín de Porres», Archivo de la Dirección General de Rehabilitación y Arquitectura, Consejería de Fomento y Vivienda de la Junta de Andalucía.

(26) Orden de la Consejería de Obras Públicas y Transportes, de 9 de febrero de 2004, por la que se declara el ámbito de actuación de San Martin de Porres de Córdoba como Rehabilitación Integral de Barriadas, Boletín Oficial de la Junta de Andalucía, $\mathrm{n}^{\circ}$ 39, 26 de febrero de 2004. 